\title{
Hybrid dynamical control based on consensus algorithm for current sharing in DC-bus microgrids
}

\author{
Carolina Albea Sanchez
}

\begin{abstract}
The main objective of this work is to propose a novel paradigm for the design of two layers of control laws for DC-bus microgrids in islanded mode. An intensive attention will be paid to the inner control level for the regulation of DC-DC electronic power converters, where the use of Hybrid Dynamical System theory will be crucial to formulate and exploit switching control signals in view of reducing the dissipated energy and improving system performance. Indeed, this recent theory is well suited for analysis of power electronic converters, since they combine continuous (voltage and currents) and discrete (on-off state of switches) signals avoiding, in this way, the use of averaged models. Likewise, an outer control level for controlling DC-bus microgrids will be developed to provide a distributed strategy that makes the microgrid scalable and robust with respect to blackouts of sources and/or loads, following the principle of $t$ Multi-Agent System theory. In this distributed strategy, they are several crucial and innovative aspects to be regarded such as the different converter architectures, the hybrid and nonlinear nature of these converters. Stability properties are guaranteed by using singular perturbation analysis.
\end{abstract}

\section{INTRODUCTION}

Microgrids linked to renewable energies emerge from the necessity to reduce the pollution as, for instance, the $\mathrm{CO}_{2}$ equivalent emissions and to account for the increasing energy demand. This class of systems are composed of distributed loads and Distributed Generations $\left(\mathrm{DG}^{1}\right)$, that can operate in parallel with the broader utility grid (grid-connected mode) with a grid-following (current dependent) control [9] or as autonomous power system (islanded mode) with a grid-forming (voltage dependent) control [28]. Moreover, they can be seen as the building blocks of Smartgrids. The main advantages of microgrids are the reliability, thanks to the DGs, their efficiency due to the reduction of the energy transmission and their ability to let to integrate alternative energy resources [20]. In order to exploit all these advantages, microgrids must ensure low distribution losses, high reliability, energy efficiency, robustness with respect to blackouts and scalability.

The interconnection between different electrical characteristics, including sources, loads and storage elements, is ensured by electronic power converters, merging to different control network structures in order to maintain

C. Albea Sanchez are with LAAS-CNRS, Univ. de Toulouse, UPS, LAAS, 7 avenue du Colonel Roche, F-31400 Toulouse, France. calbea@laas.fr

${ }^{1} \mathrm{DG}$ is an electric power generation source connected directly to the distribution network or to the customer side, thus encompasses the sources and the electronic converters [18]. microgrid power balancing. Historically, a first solution refers to the conventional droop control method [6], [21], which consists in reducing a common voltage reference for every DG, as the output current increases. This decentralized control method has been widely used for low power sharing because of its scalability and answer to plug-and-play paradigm. Nevertheless, this approach presents some important drawbacks as detailed in [15]: frequency and voltage deviations, uncertainties in the line impedances and harmonic loads.

In order to alleviate these drawbacks, some control solutions have been proposed adding a communication line between the converters, leading to two control levels: the inner control level of DGs for the conventional droop control, and outer control level of distribution bus for the extra control loop. The first control solution of these two control levels was a centralized hierarchical control. This structure provides a droop voltage (or current) reference to each DG, which is computed through the error between the measured and reference voltage (or current) of the microgrid. This method presents a good power sharing in steady state and transient time. However, it needs the communication of the outer control loop with each DG, which requires an expensive cost due to the demanded high-bandwidth communication. Moreover, it is neither robust, with respect to source blackouts and failures of the outer control loop, nor scalable. It also disregards the line resistance [14], [19], which is not suitable with the application of the plug and play paradigm and limits the potential of the approach. Another communication structure was the master/slave control, where one controlled DG specifies the current reference of the other slave DGs [7], [32]. The main advantages are the ability of recovering the outputs and its acceptable power sharing in steady state. Nevertheless, it also requires a high bandwidth communication, it is not reliable to master failures and it presents a high current overshoot in transient time, a low scalability and a reduced robustness performance. Recently, some works propose distributed control strategies with low-bandwidth communication [4], [16], [35]. These controllers are based on Multi-Agent Systems theory, proposing a leader-follower consensus control algorithm [11] in the outer control loop. By developing this algorithm by means of control theory, the microgrid can present, additionally to a low bandwidth communication, very good performance (e.g. transienttime, steady-state), reliability, efficiency, scalability and robustness. Nevertheless, this method generally disregards the nonlinear nature of power converters dynamics [22], [36]. 
The deal here focuses on providing reliable, efficient and robust distributed power flow controllers for DCmicrogrids in islanded mode. The objective consists in integrating in the DC-bus a network model for islanded mode, including the electronic converters dynamics, which are nonlinear with continuous and discrete dynamics, providing stability properties of the overall system. The main advantages of considering this more realistic dynamical model is to avoid undesirable behaviour in transient-time and to increase the energy efficiency in every loop for the considered microgrids. Hence, the loops are the following ones:

a) Inner loop: This loop is concerned by the dynamic of the microgrid power converters [4]. Converters control has been widely studied by the electronic and control communities, mostly using approximate models, such as averaged models [5], [24], [31]. These approaches have solved many practical problems in terms of theoretical justifications, but the answers still are incomplete to some extent. More recently, the control community has concentrated some efforts on the study of new hybrid control techniques applied to DC-DC converters that consider the real dynamics including: continuous-time dynamics as the current and voltage and discrete-time dynamics as the switching, for example, in [2], [10], [23] where the problem is formulated in terms of control of switched systems, whose modes are described by affine differential equations. Moreover, some solutions using this approach consider a practical implementation limitation related to avoid infinitely fast switching. A formalism relying on controlled switches, ensuring a minimum dwell time is proposed in [1], [17], [33]. The formalism used here is based on the Hybrid Dynamical Systems (HDS) theory given in [13]. This theory presents some advantages in power converters, as managing switching in the transient time for reducing the dissipated energy and avoiding current peaks in the start up [30].

b) Outer loop: The outer loop control ensures an efficient and reliable current sharing in the DC-bus in islanded mode, considering the converter dynamics. Indeed, this outer loop must generate the corresponding reference signals for each converter connected to the bus, maintaining a power balancing. The most efficient communication-based power sharing strategies are related to Multi-Agent System (MAS) theory, which basically refer to a continuous distributed interaction control law and lead the agents in the network to an agreement. The convergence properties, such as the existence of a unique equilibrium or the speed of convergence, strongly depend on the properties of communication graph among agents. This field has been very active for more than a decade and in many relevant directions have conducted to milestone results. To cite only few, one may refer to consensus algorithms, rendezvous problems, synchronization [25], [27]. Solutions consisting in the application of consensus algorithms in DC microgrids were proposed in [16] or [35]. However, none of these two publications consider the dynamics of the converters, nor ensure the network stability. The authors of [4] assess stability of ideal outer loops through an eigenvalue analysis, without regarding the dynamics of the converters. Moreover, in [8], [34] the authors propose a consensus algorithm taking into account the inner loop dynamics, controlled by sliding mode controls in [8] and by first-order filters in [34]. These dynamics are specifically focussed on buck converter architectures, which present linear average behaviours. Likewise, they neither allow a reduction of dissipated energy managing the switching, nor avoid current peaks in the start up. Moreover, the objective in [8] is a voltage balancing, which is different to the goal given here about current sharing in a DC-bus microgrid architecture and, [34] does not take into account the hybrid dynamical nature of the converters.

We follow here a different paradigm of controlling DCbus microgrids in islanded mode that gathers not only the different converter architectures, but also the switched affine model of the nodes, arising from the suggestive framework recently proposed in [12], [13]. The appealing feature of this new framework together MAS theory [27] uses Lyapunov tools to provide stability guarantees for designing distributed hybrid systems, based on a leaderfollower consensus algorithm. Moreover, time-scale separation is considered for the microgrid state variables, relating the inner loop dynamic to slow dynamics and the outer loop dynamic to fast dynamics. Hence, singular perturbation analysis in HDS [29] will provide suited stability properties. In addition, a minimum dwell time for the converter dynamics, current balancing weighted by the battery generation capacities as well as, robustness with respect to plug-in-play, connection failures and parameter variations are also ensured.

The paper is organized as follows. A characterisation of the DC-bus microgrid in islanded mode and the problem formulation is given in Section II. The mathematical model is provided in Section III. Hence, an hybrid control scheme that gathers both loops (the inner and the outer) is presented in Section IV. Stability guarantees are ensured in Section V. Likewise, some simulations are shown in Section VI validating the main result. Finally, a conclusion section closes the paper.

Notation: Throughout the paper $\mathbb{N}$ and $\mathbb{R}$ denote the set of natural and real numbers, respectively. $\mathbb{R}^{n}$ the $n-$ dimensional Euclidean space and $\mathbb{R}^{n \times m}$ the set of all real $n \times m$ matrices. $I, \mathbf{1}$ and $\mathbf{0}$ represent the identity matrix, vectors of ones and zeros, resp. of suited dimension. $\emptyset$ represents an empty set. The Euclidean norm of vector $x \in \mathbb{R}^{n}$ is denoted by $|x|$. For any symmetric matrix $M$ of $\mathbb{R}^{n \times n}$, the notation $M \succ 0(M \prec 0)$ means that the eigenvalues of $M$ are strictly positive (negative). $\operatorname{diag}\left\{a_{1}, a_{2}, \ldots, a_{N}\right\}$ is a diagonal matrix whose elements are $a_{1}, a_{2}, \ldots, a_{N} . \lambda_{m}(M)$ and $\lambda_{M}(M)$, represent the minimum and maximum eigenvalue of $M$.

\section{Formulation of THE PROBlem}

This paper considers a DC-bus microgrid composed of $L$ distributed loads, $N$ DGs composed of source units, such as, solar systems (photovoltaic and combustion), wind turbines, geothermal energy production or hydro- 
thermal plants and an integrated supercapacitor for filtering the high frequencies originated from the source units. Without loss of generality, there are $N$ batteries storing the exceeded production from the DGs, which will be returned to the grid when power consumption rises above power production. It is assumed that the source units and supercapacitors are connected to the DC-bus line through converters that optimize the power generated by the source units, as the Maximum Power Point Tracking (MPPT) converter used for photovoltaic systems and, imposing an input current to the DC-bus line, $i_{g, i}$. The $N$ batteries are connected through a converter with reversible current, $i_{b, 1}$, in order to allow the flow of power in both directions. For simplicity, we consider that the loads with their DC-DC converters, as well as, the batteries working in loading mode are Constant Power Loads (CPL), $P_{w d, p}$, with $p \in \mathcal{L}=\{1,2, . ., L\}$. As the batteries can be located far (in the space) from the DCbus line, resistances $R_{l, i}$ with $i \in \mathcal{N}=\{1,2, . ., N\}$ are added for modelling the energy dissipation due to the cable length. Note that, this assumption is not given for the sources and supercapacitors, since the $i_{g, i}$ are imposed. Likewise, there exists a power balancing over the DC-bus line, between the generated power by the DGs, $P_{w g, i}$, and the power demanded by the loads, $P_{w d, p}$. As there is a constant voltage, $V_{\text {bus }}$, in the Point of Common Coupling (PCC) of the DC-bus line, a current balancing must be ensured:

$$
\sum_{i=1}^{N} \mu_{i} i_{g, i}+\sum_{i=1}^{N}\left(\alpha_{s, i}-\alpha_{l, i}\right) i_{b, i}=\sum_{p=1}^{L} \beta_{p} i_{l, p}
$$

where $\alpha_{s, i}, \alpha_{l, i}, \beta_{p}, \mu_{i} \in\{0,1\}$. Indeed, a given battery $i$ can work in supply or loading mode, i.e., supplying current $i_{b, i}\left(\alpha_{s, i}=1\right.$ and $\left.\alpha_{l, i}=0\right)$ or demanding current $i_{b, j}\left(\alpha_{s, i}=0\right.$ and $\left.\alpha_{l, i}=1\right)$ in the DC-bus line. This balancing takes into account the plug-in-play phenomenon, that means, if a failure of any DG, battery or load is occurred, being formulated with $\mu_{i}=0$, $\alpha_{i}=\alpha_{s, i}-\alpha_{l, i}=0$ or $\beta_{p}=0$, for the DG $i$, battery $i$ or load $p$, respectively. Note that a fault mode of the battery $i$ is detected if $\alpha_{s, i}=\alpha_{l, i}$.

On the one hand, it is natural to understand that this system can be modelled by a hybrid dynamical model, because it is composed by continuous-time dynamics from the voltage and current signals and discretetime dynamics from the switching signals of the DCDC converters. On the other hand, the considered DCmicrogrid presents two control levels, as shown in Fig. 2. An inner control loop for managing the power converters of the batteries in supply mode and, an outer one, for the synchronization of the currents $i_{b, i}$, such that, robustness properties are provided to every unit with respect to any system parameter variations, and plug in play of any DG, battery or load. In the plug in play phenomenon can be also included the fact that the battery is source or load. For the sake of simplicity, it is not considered the control of the DC-DC converters of the sources, super-capacitors

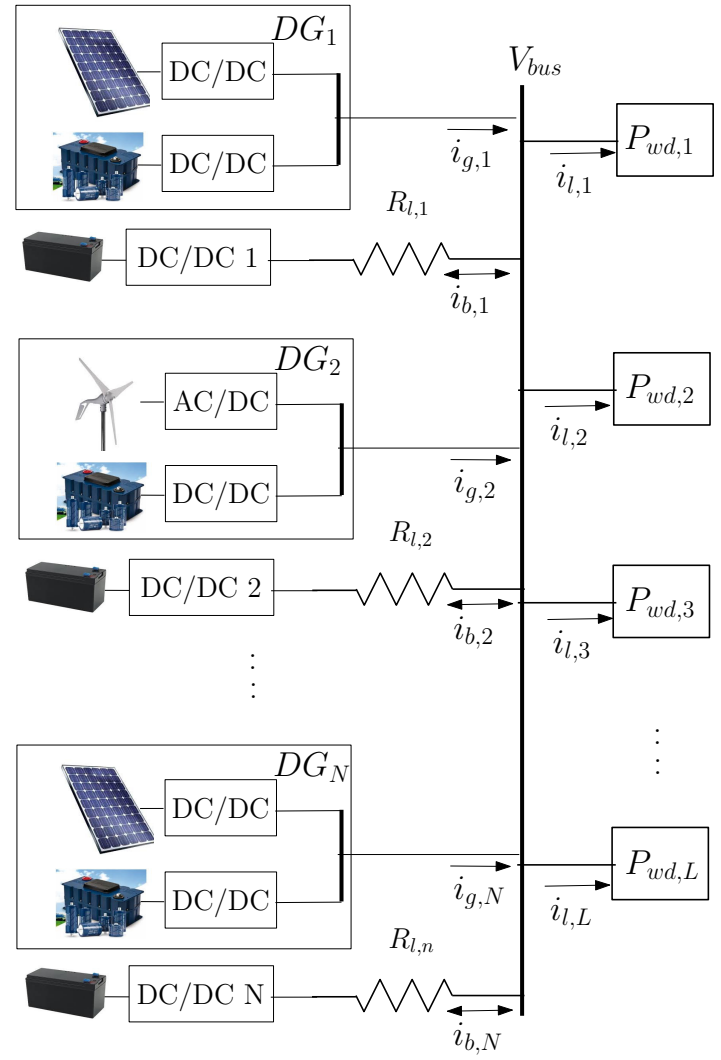

Fig. 1: Microgrid.

and loads, because

$$
\begin{aligned}
i_{g} & :=\sum_{i=1}^{N} \mu_{i} i_{g, i} \\
i_{l} & :=\sum_{p=1}^{L} \beta_{j} i_{l, p} \\
i_{b, d} & :=\sum_{j=1}^{L} \alpha_{l, i} i_{b, i}
\end{aligned}
$$

are considered exogenous inputs. Note that $i_{b, d}$ gathers the output currents of the batteries, when these ones work in loading mode. Thus, the total demanded current to be balanced by the batteries in supply mode is

$$
i_{d}:=i_{l}+i_{b, d}-i_{g} .
$$

For simplicity, let us call "node $i$ ", the $i^{t h}$ battery with its DC/DC converter and recall $x_{b, i}:=i_{b, i}$. Then, the general dynamical model of node $i$ in the microgrid is,

$$
\dot{x}_{b, i}=\chi_{i}\left(x_{b, i}, \sigma_{i}\right) \quad \text { for } \quad i \in \mathcal{N},
$$

where $x_{b, i} \in \mathbb{R}$ represents the current output of the node $i$, and $\sigma_{i} \in \mathbb{K}_{i}:=\left\{1,2, \ldots, K_{i}\right\}$ the switching of this converter, $i$.

Remark 1: It is not necessary that all nodes have access to the output currents of all nodes. Indeed, the microgrid is represented by an undirected graph $\mathcal{G}(\mathcal{N}, \mathcal{E})$, where $\mathcal{N}$ represents the nodes and $\mathcal{E}=\{1,2, . ., E\}$ the 


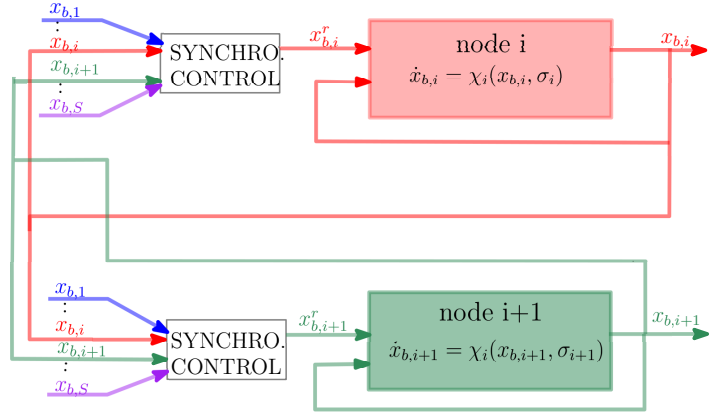

Fig. 2: Control levels in the microgrid.

interconnections between DGs. Moreover, consider that $\mathfrak{L}=\sum_{i \in \mathcal{N}_{1}} \ell_{i}$ nodes are leaders, i.e., these nodes present the information about the desired consensus value, being $\ell_{i}:\left\{\begin{array}{lll}1 & \text { if } & i \text { is leader } \\ 0 & \text { if } & i \text { is follower }\end{array}\right.$ ers. and $N-\mathfrak{L}$ nodes are follow-

The inner control level, mentioned before, concerns the design of the control law that decides over each $\sigma_{i}$. Additionally, the outer control law provides the references $x_{b, i}^{r}$ that guaranty the synchronization of all $x_{b, i}$ to a consensus value, $x_{b}^{*}$. This leader-follower consensus must not only guarantee the consensus between all references $x_{b, i}^{r}$, but also the current balancing given in (1). Hence, the following definition

$$
x_{b}^{*}:=i_{d} / \sum_{i=1}^{N} \alpha_{s, i},
$$

which will be the desired current reference for the $\mathfrak{L}$ leaders.

The problem considered here is formulated as follows:

Problem 1: Design a hybrid dynamical system for (3), that considers both the continuous-time and discretetime dynamics of each node $i$, providing

1) a control law for the switching signal $\sigma_{i}$, that guaranties a globally asymptotically stability of a small neighbourhood of $x_{b, i}$,

2) and a control law for the continuous-time signal $x_{b, i}^{r}$, that ensures an asymptotic stability of all $x_{b, i}^{r}$ to a neighbourhood of $x_{b}^{*}$ defined in (4),

guaranteeing a robust system w.r.t., parameter variations and plug in play of the DGs, nodes and loads.

This problem considers a current balancing between all $x_{b, i}$ having every node the same weight. Nevertheless, later, in Section V a current balancing with nodes weighted by battery generation capacities will be provided.

\section{Modelling OF A DC MICROGRID}

First, let us focus in a node $i$ and its connection with the DC-bus line, as depicted in Fig. 3. The dynamical model of this node $i$ can be rewritten as follows:

$$
\begin{aligned}
\dot{x}_{i} & =A_{\sigma, i} x_{i}+B_{\sigma, i} \\
y_{i} & =C_{\sigma, i} x_{i}
\end{aligned}
$$

where $A_{\sigma, i}, B_{\sigma, i}$ and $C_{\sigma, i}$ are matrices of suited dimensions, $\sigma_{i}$ is the input control signal which switches in $\mathbb{K}_{i}$,

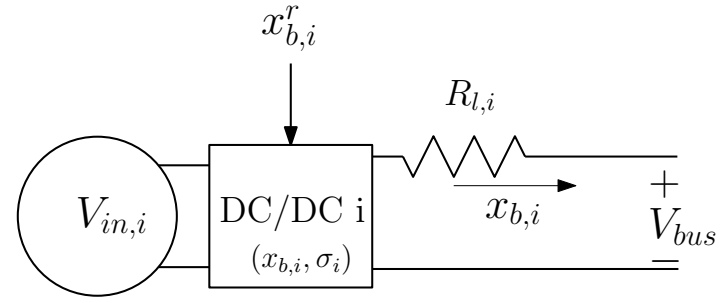

Fig. 3: Node $i$ in the microgrid.

$y_{i} \in \mathbb{R}^{n_{i}}$ is the output system and $x_{i}=\left[x_{\gamma, i}, x_{\varrho, i}\right]^{\top} \in \mathbb{R}^{n_{i}}$ is the system state, being $x_{\gamma, i} \in \mathbb{R}^{1}$ a current signal through any inductance and $x_{\varrho, i} \in \mathbb{R}^{n_{i}-1}$ a vector with other state signals.

For one side, it is easy to see that the reference $x_{b, i}^{r}$ provides the equilibrium of current $x_{\gamma, i}$, through

$$
x_{e, \gamma, i}=\phi_{i}\left(x_{b, i}^{r}\right),
$$

being $\phi_{i}$ a function given by the converter architecture. On the other side, the equilibrium of (5), $x_{e, i}:=$ $\left[x_{e, \gamma, i} x_{e, \varrho, i}\right]$, not necessarily has to be the solution of the continuous-time system, but it can be the solution of the switching system, leading to a generalized solution in sense of Fillipov, as given in [2], [10]. The set of equilibriums of system (5) is characterized by the following sufficient condition.

Assumption 1: For system (5), there exists $\lambda_{i}=$ $\left[\lambda_{i, 1}, \lambda_{i, 2}, \ldots, \lambda_{i, N}\right]$ satisfying $\sum_{k=1}^{N} \lambda_{i, k}=1$ and such that the following convex combination holds

$$
\sum_{i, k}^{S} \lambda_{i, k}\left(A_{k, i} x_{i}+B_{k, i}\left(x_{b, i}\right)\right) \text {. }
$$

Moreover, the power converters fulfil the following property.

Property 1: Given matrices $A_{k, i}$ for system (5), with $k \in \mathbb{K}_{i}$, then there exists matrices $P_{i} \succ 0 \in \mathbb{R}^{n_{i}}$ and $Q_{i} \succ 0 \in \mathbb{R}^{n_{i}}$, such that,

$$
A_{i, k}^{\top} P_{i}+P_{i} A_{i, k} \prec-2 Q_{i},
$$

for all $k \in \mathbb{K}_{i}$.

This property requires that every $A_{i, k}$ is Hurwitz.

\section{HYBRID CONTROL STRUCTURE}

This section is devoted to propose a hybrid dynamical structure for the mentioned two control levels, following the paradigm given in [13], and such that considers the continuous-time and discrete-time dynamics and ensures all items of Problem 1. The overall dynamics is represented as follows:

$$
\mathcal{H}_{i}\left(\xi_{i}, x_{b}^{r}\right):\left\{\begin{array}{l}
{\left[\begin{array}{c}
\dot{x}_{i} \\
\dot{\sigma}_{i} \\
\dot{\tau}_{i} \\
\dot{x}_{b, i}^{r}
\end{array}\right]=f_{i}\left(\xi_{i}, x_{b}^{r}\right) \quad\left(\xi_{i}, x_{b, i}^{r}\right) \in \mathcal{C}_{i},} \\
{\left[\begin{array}{l}
x_{i}^{+} \\
\sigma_{i}^{+} \\
\tau_{i}^{+} \\
x_{b, i}^{r+}
\end{array}\right] \in G_{i}\left(\xi_{i}, x_{b}^{r}\right) \quad\left(\xi_{i}, x_{b, i}^{r}\right) \in \mathcal{D}_{i},}
\end{array}\right.
$$


where $\xi_{i}:=\left[x_{i}, \sigma_{i}, \tau_{i}\right]^{\top}$ and $x_{b}^{r}:=\left[x_{b, 1}^{r}, x_{b, 2}^{r}, . ., x_{b, N}^{r}\right]^{\top}$. Likewise, $\sigma_{i}^{+} \in \mathbb{K}_{i}$ and $\dot{x}_{b, i}^{r} \in \mathbb{R}$ are designed for satisfying the inner and outer control objectives of Problem 1 , respectively. Finally, $f_{i}$ is a map that defines the continuous-time dynamic and $G_{i}$ is a (set-valued) map that captures the switching logic. Both of them, are defined as follows:

$$
f_{i}:=\left[\begin{array}{c}
\alpha_{s, i}\left(A_{\sigma, i} x_{i}+B_{\sigma, i}\right) \\
0 \\
r\left(\frac{\tau_{i}}{T_{i}}\right) \\
-\alpha_{s, h} K_{c, i}\left(\sum_{j=1}^{N}\left(x_{b, i}^{r}-x_{b, j}^{r}\right)+\tilde{x}_{b, i}^{r} \ell_{i}\right)-\alpha_{s, i}\left(x_{\gamma, i}-x_{e, \gamma, i}\right)
\end{array}\right]
$$

$G_{i} \in\left[\begin{array}{c}x_{i} \\ \underset{\sigma \in \mathbb{K}}{\operatorname{argmin}}\left(x_{i}-x_{e, i}\right)^{\top} P_{i} \alpha_{s, i}\left(A_{\sigma, i} x_{i}+B_{\sigma, i}\right) \\ 0 \\ \tilde{x}_{b, i}^{r}\end{array}\right]$,

such that, $f_{i}, G_{i} \in \mathbb{H}_{i}:=\mathbb{R}^{n_{i}} \times \mathbb{K}_{i} \times\left[0,2 T_{i}\right] \times \mathbb{R}^{1}, K_{c, i}$ a positive parameter to be designed and $\tilde{x}_{b, i}^{r}:=x_{b, i}^{r}-x_{b}^{*}$. Note that $x_{b}^{*}$, given in (4), is a picewise function that can change from the exogenous discrete variables, $\mu_{i}, \alpha_{s, i}$, $\alpha_{l, i}$ and $\beta_{j} \cdot r\left(\frac{\tau_{i}}{T_{i}}\right)=\min \left(1,2-\frac{\tau_{i}}{T_{i}}\right)$, with $T_{i}$ a positive parameter that fixes the minimum dwell time. Likewise, $x_{e, i}$ is related to $x_{b, i}^{r}$ from (6). The so-called "flow" and "jump" sets $\mathcal{C}_{i}$ and $\mathcal{D}_{i}$, respectively, are given by

$$
\begin{aligned}
\mathcal{C}_{i}: & =\left\{\left\{\xi_{i} \in \mathbb{H}_{i}: \tilde{x}_{i}^{\top} P_{i} \alpha_{s, i}\left(A_{\sigma, i} x_{i}+B_{\sigma, i}\right) \leq-\alpha_{s, i} \eta_{i} \tilde{x}_{i}^{\top} Q_{i} \tilde{x}_{i}\right\}\right. \\
& \left.\cup\left\{\xi_{i}: \tau_{i} \in\left[0, T_{i}\right]\right\}\right\} \times R \mathbb{B}_{i} \\
\mathcal{D}_{i}: & =\left\{\left\{\xi_{i} \in \mathbb{H}_{i}: \tilde{x}_{i}^{\top} P_{i} \alpha_{s, i}\left(A_{\sigma, i} x_{i}+B_{\sigma, i}\right) \geq-\alpha_{s, i} \eta_{i} \tilde{x}_{i}^{\top} Q_{i} \tilde{x}_{i}\right\}\right. \\
& \left.\cap\left\{\xi_{i}: \tau_{i} \in\left[T_{i}, 2 T_{i}\right]\right\}\right\} \times R \mathbb{B}_{i},
\end{aligned}
$$

where $\tilde{x}_{i}:=x_{i}-x_{e, i}$ and $R \mathbb{B}_{i}$ a closed ball of radius $R$ in the Euclidean norm centred at the origin, thus it is compact underlying set of $\left\{\xi_{i} \in \mathbb{H}_{i}: \tilde{x}_{i}^{\top} P_{i} \alpha_{s, i}\left(A_{\sigma, i} x_{i}+B_{\sigma, i}\right) \leq-\alpha_{s, 1} \eta_{i} \tilde{x}_{i}^{\top} Q_{i} \tilde{x}_{i}\right\} \quad$ where $x_{b, i}^{r}$ evolves.

Note that parameter $\alpha_{s, i}$ decides if the state $x_{i}$ of node $i$ evolves according dynamic (5), supplying current $x_{b, i}$ to the DC-bus. If this parameter switches to loading mode (i.e. $\alpha_{s, i}=0, \alpha_{l, i}=1$ ), the dynamic of $x_{i}$ will be $\dot{x}_{i}=0$. However, the demanded current, $i_{d}$, defined in (2) will change, and consequently, $x_{b}^{*}$, the reference signals $x_{b, h}^{r}$ and the desired equilibrium $x_{e, h}, \forall h \neq i$ will be also modified. This variation in $i_{d}$, and thus in $x_{e, h}$ occurs, if any node, any DG or any load fails or quickly changes, because the microgrid elements are connected by $x_{b, h}^{r}$. Moreover, parameter $\eta_{i} \in(0,1)$ manages a tradeoff in the transient time between reducing the switching frequency and improving any performance level given by a functional, as shown in [1], [2]. Let us remind that the advantages of using HDS for controlling power converters are shown in [30]. Finally, it is emphasized, that $\mathcal{H}_{i}$ defines an event-triggered control that guarantees a minimum dwell time for each node $i$ given by $T_{i}$. Indeed, all solutions of $\mathcal{H}_{i}$ must flow at least $T_{i}$ ordinary time after each jump, because the dynamic $\dot{\tau}_{i}=1$ evolves, while $\tau_{i} \leq T_{i}$.
The characterized system $\mathcal{H}_{i}$ allows introducing the following compact attractor

$$
\mathcal{A}_{i}:=\left\{\xi_{i} \in \mathbb{H}_{i}: V_{i}\left(\tilde{x}_{i}\right)<\varepsilon_{i}\right\} \times R \mathbb{B}_{i} .
$$

where

$$
\begin{gathered}
V_{i}\left(\tilde{x}_{i}\right)=\max \left\{W_{i}\left(\tilde{x}_{i}\right)-\varepsilon_{i}, 0\right\} \\
W_{i}\left(\tilde{x}_{i}\right):=\frac{\tilde{x}_{i}^{T} P_{i} \tilde{x}_{i}}{2}
\end{gathered}
$$

with

and $P_{i} \succ \in \mathbb{R}^{n_{i}}$.

It is emphasized that the minimum dwell time, $T_{i}$, leads to the convergence of $x_{i}$ to a neighbourhood of $x_{e, i}$, being the distance between $x_{i}$ to $x_{e, i}$ characterized by the upper bound $\varepsilon_{i}$. Next lemma is invoked, in order to establish a property that defines the relationship between the upper bounds of $\varepsilon_{i}$ and $T_{i}$ in the stead state.

Lemma 1: [3, Lemma 2] Consider that Assumption 1 and Property 1 are satisfied, then all the eigenvalues of the matrix $P_{i}^{-1} Q_{i}$ are positive and $\left\|e^{A_{i, k} t}\right\| \leq$ $\frac{\lambda_{i, m}^{-1 / 2}\left(P_{i}\right)}{\lambda_{i, M}^{-1 / 2}\left(P_{i}\right)} e^{-\alpha_{i} t}$, where $\alpha_{i}:=\lambda_{m}\left(P_{i}^{-1} Q_{i}\right)$.

Then, a practical minimum dwell time can be established.

Property 2: There exists a positive scalar $T_{i}^{*}$ and an associated $\varepsilon_{i}^{*}=\varepsilon_{i}\left(T_{i}^{*}\right)$, such that for any chosen $T_{i} \leq$ $T_{i}^{*}$, inducing $\varepsilon_{i} \leq \varepsilon_{i}^{*}$, the solutions $(t, j) \in\left[t_{s s}, \infty\right) \times$ $\left[j_{s s}, \infty\right) \rightarrow \varphi(t, j)$ to hybrid system $\mathcal{H}_{i}$ flow in the steady state for at least $T_{i}$ ordinary time after the jump, $j$ and before reaching set $\mathcal{D}_{i}$.

Proof: We follow the proof of [3, Property 1]. Then, consider the first jump in the steady state $\left(t_{j}, j\right) \in$ $\left[t_{s s}, \infty\right) \times\left[j_{s s}, \infty\right) \rightarrow \varphi\left(t_{j}, j\right)$ to hybrid system $\mathcal{H}_{i}$ allowing to introduce the variable $\tilde{t}=t-t_{j}$. From the instant time $\left(t_{j}, j\right)$, the solution for node $i$ flows in the set $\mathcal{C}_{i}$, being the trajectories described by

$$
\dot{\tilde{x}}_{i}(\tilde{t})=A_{\sigma, i} \tilde{x}_{i}(\tilde{t})+b_{\sigma, i}, \quad \tilde{x}_{i}\left(t_{j}\right)=\tilde{x}_{i, j},
$$

with $b_{\sigma, i}:=B_{\sigma, i}-A_{\sigma, i} x_{e, i}(\tilde{t})$. Then, it is had

$$
\tilde{x}_{i}(\tilde{t})=e^{A_{\sigma, i} \tilde{t}}\left(\tilde{x}_{i, j}+A_{\sigma, i}^{-1} b_{\sigma, i}\right)-A_{\sigma, i}^{-1} b_{\sigma, i},
$$

for $0 \leq \tilde{t} \leq T_{i}$. From the properties of a norm, it is got

$$
\left\|\tilde{x}_{i}(\tilde{t})+A_{\sigma, i}^{-1} b_{\sigma, i}\right\|=\left\|e^{A_{\sigma, i} \tilde{t}}\right\|\left\|\tilde{x}_{i, j}+A_{\sigma, i}^{-1} b_{\sigma, i}\right\|
$$

and applying Lemma 1 , it is had

$\left\|\tilde{x}_{i}(\tilde{t})+A_{\sigma, i}^{-1} b_{\sigma, i}\right\|=\frac{\lambda_{i, m}^{-1 / 2}\left(P_{i}\right)}{\lambda_{i, M}^{-1 / 2}\left(P_{i}\right)} e^{-\alpha_{i} t}\left\|\tilde{x}_{i, j}+A_{\sigma, i}^{-1} b_{\sigma, i}\right\|$.

Then, following the reasoning in [3, Property 1], the upper bound of dwell time $T_{i}$ is

$$
T_{i}^{*}=\min _{\sigma} \frac{1}{\alpha_{i}} \operatorname{Ln}\left(\frac{\lambda_{i, M}^{-1 / 2}\left(P_{i}\right)\left\|\tilde{x}_{i, j}+A_{\sigma, i}^{-1} b_{\sigma, i}\right\|}{\lambda_{i, m}^{-1 / 2}\left(P_{i}\right)\left\|\tilde{x}_{i, j}\left(T_{i}^{*}\right)+A_{\sigma, i}^{-1} b_{\sigma, i}\right\|}\right),
$$

such that, $0 \leq T_{i} \leq T_{i}^{*}$ and $\tilde{x}_{i, j}\left(T_{i}^{*}\right) \in$ $\left\{-\frac{2 \varepsilon_{i}^{*}}{\lambda_{M, i}\left(P_{i}\right)}, \frac{2 \varepsilon_{i}^{*}}{\lambda_{M, i}\left(P_{i}\right)}\right\}$. Note that the mode $\sigma$ is given by the set-valued map $G_{i}$. 
Therefore, it is concluded that the maximum admissible chattering in the steady state, $(t, j) \in\left[t_{s s}, \infty\right) \times$ $\left[j_{s s}, \infty\right)$, induced by $T_{i}^{*}$ is defined from $\tilde{x}_{i, j}$ and $\tilde{x}_{i, j}\left(T_{i}^{*}\right)$, for node $i \in \mathcal{N}$.

Remark 2: It is easy to see, from (14), that if $\varepsilon_{i}^{*}$ goes to zero, then $T_{i}^{*}$ goes to $\frac{1}{\alpha_{i}} \operatorname{Ln}\left(\frac{\lambda_{i, M}^{-1 / 2}\left(P_{i}\right)}{\lambda_{i, m}^{-1 / 2}\left(P_{i}\right)}\right)$. It should be expected that if $\varepsilon_{i}^{*}$ goes to zero, then $T_{i}^{*}$ also goes to zero, however the conservatism introduced in Lemma 1 avoids to reach this intuitive result.

\section{A. Global hybrid structure}

Highlighting that the microgrid is composed of $i \in \mathcal{N}$ subsystems, the idea is to control locally every one of these $i$-subsystem, in such a way that the global system enjoys of suited stability properties. In order to define a compact hybrid system that gathers all dynamics, it is needed to introduce next definition about the Laplacian matrix.

Definition 1: The Laplacian matrix represents the undirected graph $\mathcal{G}(\mathcal{L}, \mathcal{E})$ of interconnections given by: $L\left(\alpha_{s}\right)=\Delta\left(\alpha_{s}\right)-\mathcal{A}\left(\alpha_{s}\right)$, being $\alpha_{s}:=\operatorname{diag}\left\{\alpha_{s, 1}, \alpha_{s, 2}, . ., \alpha_{s, N}\right\}, \mathcal{A}\left(\alpha_{s}\right)$ the adjacency matrix $\mathcal{A}\left(\alpha_{s}\right)=\left[a_{i h}\left(\alpha_{s}\right)\right]$, where

$$
a_{i h}\left(\alpha_{s}\right):\left\{\begin{array}{ccc}
\alpha_{s, i} \alpha_{s, h} & \text { if } & i \neq h \\
0 & \text { if } & i=h
\end{array} \forall i, h \in \mathcal{N}\right.
$$

and the diagonal matrix $\Delta\left(\alpha_{s}\right)=\left[\delta_{i h}\left(\alpha_{s}\right)\right]$, with

$$
\delta_{i h}\left(\alpha_{s}\right):\left\{\begin{array}{ccc}
0 & \text { if } & i \neq h \\
\sum_{p \in \mathcal{N}, p \neq i} a_{i p}\left(\alpha_{s}\right) & \text { if } & i=h
\end{array} \forall i, h \in \mathcal{N} .\right.
$$

Note that the Laplacian matrix $L\left(\alpha_{s}\right)$ is a symmetric positive semi-definite matrix [26].

This notation allows to define the global hybrid system

$$
\mathcal{H}\left(\xi, x_{b}^{r}\right):\left\{\begin{array}{l}
{\left[\begin{array}{l}
\dot{x} \\
\dot{\sigma} \\
\dot{\tau} \\
\dot{x}_{b}^{r}
\end{array}\right]=f\left(\xi, x_{b}^{r}\right) \quad\left(\xi, x_{b}^{r}\right) \in \mathcal{C},} \\
{\left[\begin{array}{l}
x^{+} \\
\sigma^{+} \\
\tau^{+} \\
x_{b}^{r+}
\end{array}\right] \in G\left(\xi, x_{b}^{r}\right) \quad\left(\xi, x_{b}^{r}\right) \in \mathcal{D},}
\end{array}\right.
$$

such that,

$$
\begin{aligned}
f\left(\xi, x_{b}^{r}\right) & :=\left[\begin{array}{c}
A_{\sigma} x+B_{\sigma} \\
0 \\
r \\
-K_{c}\left(L\left(\alpha_{s}\right) x_{b}^{r}+\alpha_{s} \mathcal{I}_{\ell} \tilde{x}_{b}^{r}\right)-\alpha_{s}\left(x_{\gamma}-x_{e, \gamma}\right)
\end{array}\right] \\
G\left(\xi, x_{b}^{r}\right) & =\bigcup_{i=1,2, . . N} G_{i}\left(\xi_{i}, x_{b, i}^{r}\right)
\end{aligned}
$$

being

$$
\begin{aligned}
x & :=\left[x_{1}, x_{2}, . ., x_{N}\right]^{\top} \\
\sigma & :=\left[\sigma_{1}, \sigma_{2}, . ., \sigma_{N}\right]^{\top} \\
\tau & :=\left[\tau_{1}, \tau_{2}, . ., \tau_{N}\right]^{\top} \\
r & :=\left[r_{1}\left(\frac{\tau_{i}}{T_{i}}\right), r_{2}\left(\frac{\tau_{2}}{T_{2}}\right), . . r_{N}\left(\frac{\tau_{N}}{T_{N}}\right)\right]^{\top} \\
\mathcal{I}_{\ell}\left(\ell_{i}\right) & :=\operatorname{diag}\left\{\ell_{1}, \ell_{2}, . ., \ell_{N}\right\} \\
\tilde{x}_{b}^{r} & :=\left[\tilde{x}_{b, 1}^{r}, \tilde{x}_{b, 2}^{r}, . ., \tilde{x}_{b, N}^{r}\right]^{\top} \\
x_{\gamma} & :=\left[x_{\gamma, 1}, x_{\gamma, 2}, . ., x_{\gamma, N}\right]^{\top} \\
x_{e, \gamma} & :=\left[x_{e, \gamma, 1}, x_{e, \gamma, 2}, . ., x_{e, \gamma, N}\right]^{\top}, \\
A_{\sigma} & :=\operatorname{diag}\left\{\alpha_{s, 1} A_{\sigma, 1}, \alpha_{s, 2} A_{\sigma, 2}, . ., \alpha_{s, N} A_{\sigma, N}\right\} \\
B_{\sigma} & :=\left[\alpha_{s, 1} B_{\sigma, 1}, \alpha_{s, 2} B_{\sigma, 2}, . ., \alpha_{s, N} B_{\sigma, N}\right]^{\top} \\
K_{c} & :=\operatorname{diag}\left\{\alpha_{s, 1} K_{c, 1}, \alpha_{s, 2} K_{c, 2}, . ., \alpha_{s, N} K_{c, N},\right\}
\end{aligned}
$$

and

$$
\mathcal{C}=\bigcup_{i=1,2, . . N} \mathcal{C}_{i}, \quad \mathcal{D}=\bigcup_{i=1,2, \ldots N} \mathcal{D}_{i}
$$

Now, from this hybrid model of the complete microgrid, the main result of this work can be presented.

\section{MAIN RESULT}

According to Property 2, the main objective is to guarantee that $x_{i}$ converges to a neighbourhood of $x_{e, i}$, such that, $\left|x_{i}-x_{e, i}\right|<\frac{2 \varepsilon_{i}^{*}}{\lambda_{M, i}\left(P_{i}\right)}$, and that all $x_{b, i}^{r}$ converge to a consensus defined by $x_{b}^{*}(4)$, such that, $\left|x_{b, i}^{r}-x_{b}^{*}\right|<$ $\psi_{i}\left(\varepsilon_{i}\right)$, being $\psi_{i}\left(\varepsilon_{i}\right)$ a function given by the converter architecture, which relates the state, $\xi_{i}$ and $x_{b, i}$.

Next lemma is introduced in order to prove the main result.

Lemma 2: For a given $P_{i}$ and $Q_{i}$ satisfying Property 1 , System 5 presents the following property

$$
\min _{k \in \mathbb{K}} \tilde{x}_{i}^{\top} P_{i} \alpha_{s, i}\left(A_{k, i} x_{i}+B_{k, i}\right) \prec-\eta_{i} \alpha_{s, i} \tilde{x}_{i}^{T} Q_{i} \tilde{x}_{i}
$$

Proof: The proof is easily deducted from [2, Theorem 1].

Now, let us focus in the hybrid system $\mathcal{H}$ which is composed of slow variables, $\xi$, and fast continuous-time variables, $x_{b}^{r}$. Thus, the inner control level, $\sigma$, manages the slow variables and the outer control level, $x_{b}^{r}$, manages the fast variables. Moreover, the tuned parameters $K_{c, i}$ are selected for ensuring the next:

Assumption 2: Take

$$
K_{c, i}>>\frac{3}{T_{i}}
$$

such that, $x_{b, i}^{r}$ converges very fast to a consensus with respect to the ordinary time evolution.

Therefore, let us rewrite $\mathcal{H}$ in a singular perturbation 
form:

$$
\mathcal{H}_{s p}\left(\xi, x_{b}^{r}\right):\left\{\begin{array}{l}
{\left[\begin{array}{l}
\dot{x} \\
\dot{\sigma} \\
\dot{\tau} \\
\nu \dot{x}_{b}^{r}
\end{array}\right]=f_{s p}\left(\xi, x_{b}^{r}\right) \quad\left(\xi, x_{b}^{r}\right) \in \mathcal{C},} \\
{\left[\begin{array}{l}
x^{+} \\
\sigma^{+} \\
\tau^{+} \\
x_{b}^{r+}
\end{array}\right] \in G\left(\xi, x_{b}^{r}\right) \quad\left(\xi, x_{b}^{r}\right) \in \mathcal{D},}
\end{array}\right.
$$

such that,

$$
f_{s p}(\xi):=\left[\begin{array}{c}
A_{\sigma} x+B_{\sigma} \\
0 \\
r \\
-\left(L\left(\alpha_{s}\right) x_{b}^{r}+\alpha_{s} \mathcal{I}_{\ell} \tilde{x}_{b}^{r}\right)-\nu \alpha_{s}\left(x_{\gamma}-x_{e, \gamma}\right)
\end{array}\right]
$$

where $\nu:=K_{c}^{-1}>0$ is small enough ensuring Assumption 2. Indeed, the outer control level, $x_{b}^{r}$, evolves sufficiently fast with respect to the inner control level $\sigma$ evolution, such that, a regulation problem for $x$ can be considered. Note that the jump set $G$ in a singular perturbed form does not change.

An analysis of the singular perturbation regularization given in (22) is leaded in order to stablish Semi Gmobal Practical Stability (SPAS) as $\nu$ goes to $0^{+}$of set $\mathcal{A}:=$ $\mathcal{A}_{1} \cup \mathcal{A}_{2} \cup \ldots \cup \mathcal{A}_{N}$. For this deal, let us define the suited properties stated in the following propositions.

Proposition 1: System $\mathcal{H}_{s p}\left(f_{s p}, G, \mathcal{C}, \mathcal{D}\right)$ is well-posed.

Proof: It is easy to see that hybrid system $\mathcal{H}_{s p}\left(f_{s p}, G, \mathcal{C}, \mathcal{D}\right)$ verifies the following properties

- $\mathcal{C}$ and $\mathcal{D}$ are closed sets in $\mathbb{H}:=\bigcup_{i=1, \ldots, N} \mathbb{H}_{i}$;

- $f_{s p}$ is a continuous function, thus locally bounded and outer semi-continuous. Moreover, it is convex for each $\left(\xi, x_{b}^{r}\right) \in \mathcal{C}$;

- $G$ is outer semi-continuous and locally bounded.

Proposition 2: Regularity of the system $\mathcal{H}_{s p}$ "manifold".

Proof: The set-valued mapping $\mathcal{M}: \mathbb{H} \backslash \mathbb{R}^{N} \rightarrow \mathbb{R}^{N}$ is a "manifold", i.e., it is a map between the slow variable $\xi \in \mathbb{H} \backslash \mathbb{R}^{N}$ and the fast variable $x_{b}^{r} \in \mathbb{R}^{N}$ and corresponds to the quasi-steady-state equilibrium manifold in singular perturbation theory, i.e. as $\nu \rightarrow \mathbf{0}^{+}$, it is got $\mathcal{I}_{\ell} x_{b}^{r} \rightarrow x_{b}^{*} \mathbf{1}$ and $L\left(\alpha_{s}\right) x_{b}^{r} \rightarrow \mathbf{0}$ to get a consensus. Here, this manifold is defined as follows:

$$
\mathcal{M}(\xi)= \begin{cases}-\nu\left(x_{\gamma}-x_{e, \gamma}\right) & \xi \in \mathcal{C} \backslash R \mathbb{B} \\ \emptyset & \xi \notin \mathcal{C} \backslash R \mathbb{B} .\end{cases}
$$

Remind that $x_{e, \gamma}$ depends on $x_{b}^{r}$ according to (6). Readily, it is seen that this map is locally bounded and outer semi-continuous. Unlike, $\mathcal{M}(\xi)$ is a nonempty subset of $R \mathbb{B}$, for each $\xi \in \mathcal{C}$.
Proposition 3: Consider the reduced system given by

$$
\mathcal{H}_{r}(\xi): \begin{cases}{\left[\begin{array}{c}
\dot{x} \\
\dot{\sigma} \\
\dot{\tau}
\end{array}\right]=\left[\begin{array}{c}
A_{\sigma} x+B_{\sigma} \\
0 \\
r
\end{array}\right] \quad \xi \in \mathcal{C}(\mathcal{M}),} \\
{\left[\begin{array}{l}
x^{+} \\
\sigma^{+} \\
\tau^{+}
\end{array}\right] \in G_{r}(\xi)} & \xi \in \mathcal{D}(\mathcal{M}),\end{cases}
$$

being $G_{r}(\xi):=\bigcup_{i=1,2, \ldots N} G_{r, i}\left(\xi_{i}\right)$ with $G_{r, i}\left(\xi_{i}\right):=$ $\left[\begin{array}{c}x_{i} \\ \underset{\sigma \in \mathbb{K}}{\operatorname{argmin}}\left(x_{i}-x_{e, i}\right) \top P_{i} \alpha_{s, i}\left(A_{\sigma, i} x_{i}+B_{\sigma, i}\right) \\ 0\end{array}\right]$.

Then, $\mathcal{A}_{r}:=\mathcal{A} \backslash R \mathbb{B}$ with $R \mathbb{B}:=R \mathbb{B}_{1} \cup R \mathbb{B}_{2} \cup . . \cup R \mathbb{X} \mathbb{B}_{N}$ is Uniformly Globally Asymptotically Stable (UGAS).

Proof: Let us consider the following candidate Lyapunov function for the reduced hybrid system,

$$
V(\tilde{x})=\max \{W(\tilde{x})-\varepsilon(T)\}
$$

with

$$
W(\tilde{x}):=\frac{\tilde{x}^{T} P \tilde{x}}{2},
$$

$P=\operatorname{diag}\left\{P_{1}, P_{2}, . ., P_{N}\right\}$ and $\varepsilon(T)$ the associated parameter to $T=: \max \left(T_{i}\right)$, such that $T_{i}<T_{i}^{*}$ for each $i \in \mathcal{N}$, as given in Property 2, We emphasize that $W(\tilde{x})$ may increase for $\tau_{i} \in\left[0, T_{i}\right]$, because the solutions can be forced to flow a maximum ordinary time $T$.

Next, from Lemma 2, the affine definition of $\dot{W}(\tilde{x})$ and the reasoning given in $[1$, Section V.B], the increase of $W(\tilde{x})$ can be bounded by

$$
W(\tilde{x}) \leq \varepsilon(T):=2 e^{2 \kappa_{1} T} \varepsilon\left(T^{*}\right)+\frac{2 \kappa_{2}^{2}}{\kappa_{1}^{2}}\left(e^{\kappa_{1} T}-1\right)^{2},
$$

for all $0<\tau_{i}<T$, being $\kappa_{1}$ and $\kappa_{2}$ positive parameters that allow to bound $|\tilde{x}|$. Hence, if $T$ shrinks to zero, then $\varepsilon(T)$ diminishes.

Now, let us introduce the following compact set

$$
\mathcal{A}_{\mathcal{E}}:=\left\{\xi \in \mathbb{H} \backslash \mathbb{R}^{N}: V(\tilde{x})<\varepsilon\right\} .
$$

It is easy to see that $V$ is continuous in $\mathcal{C} \cup \mathcal{D}$ and locally Lipschitz near each point in $\mathcal{C} \backslash \mathcal{A}_{\mathcal{E}}$. Likewise, $V$ is positive definite with respect to $\mathcal{A}_{\varepsilon}$ in $\mathcal{C} \cup \mathcal{D}$ and radially unbounded. Then, UGAS proof of $\mathcal{A}_{\mathcal{E}}$ for the reduced hybrid system $\mathcal{H}_{r}$ is complete from Lemma 2 and $[1$, Theorem 3].

The selection of $T$ and its associated $\varepsilon$ allows achieving $\mathcal{A}_{r} \subset \mathcal{A}_{\mathcal{E}}$. Therefore, if $\mathcal{A}_{\mathcal{E}}$ is UGAS for $\mathcal{H}_{r}(\xi)$, then $\mathcal{A}_{r}$ is UGAS, as well.

Proposition 4: Consider the "boundary layer"

$$
\left[\begin{array}{c}
\dot{x} \\
\dot{\sigma} \\
\dot{\tau} \\
\dot{x}_{b}^{r}
\end{array}\right]=\left[\begin{array}{c}
0 \\
0 \\
0 \\
-\left(L\left(\alpha_{s}\right) x_{b}^{r}+\alpha_{s} \mathcal{I}_{l} \tilde{x}_{b}^{r}\right)-\alpha_{s}\left(x_{\gamma}-x_{e, \gamma}\right)
\end{array}\right],
$$


such that, $\left(\xi, x_{b}^{r}\right) \in(\mathcal{C} \backslash R \mathbb{B} \cap \rho \mathbb{B}) \times R \mathbb{B}$, for any $\rho>0$ large enough. Then, the compact set

$$
\mathcal{M}_{\rho}:=\left\{\left(\xi, x_{b}^{r}\right): \xi \in(\mathcal{C} \backslash R \mathbb{B} \cap \rho \mathbb{B}), x_{b}^{r} \in R \mathbb{B}\right\}
$$

is globally asymptotically stable.

Proof: Readily, it is seen that the flow set in the boundary layer given in (26) is compact. Moreover, the dynamic (26) are obtained re-scaling the ordinary time, $t$, in $(22)$, by $1 / \nu$. Unlike, there is not jump in the boundary layer if (21) is satisfied, remaining $\xi$ constant during flows.

Consider $\rho=\rho_{1}$ and $\rho=\rho_{2}$, such that, $0<\rho_{1}<\rho_{2}$. As $\mathcal{M}_{\rho_{1}} \subset \mathcal{M}_{\rho_{2}}$, if $\mathcal{M}_{\rho_{2}}$ is globally asymptotically stable for (26), then $\mathcal{M}_{\rho_{1}}$ also is globally asymptotically stable. Then, as $\mathcal{C} \backslash R \mathbb{B}$ is compact, it is had $\mathcal{C} \backslash R \mathbb{B} \cap \rho \mathbb{B}=\mathcal{C} \backslash R \mathbb{B}$ for all $\rho$ large enough.

Now, consider the particular solution of $\xi$ in the boundary layer, such that, $x-x_{e}=0$, that means, $x_{\gamma}=x_{e, \gamma}$. Therefore, if it is chosen

$$
\begin{aligned}
V_{c} & :=\frac{1}{2}\left(\tilde{x}_{b}^{r \top} L\left(\alpha_{s}\right) x_{b}^{r}+\tilde{x}_{b}^{r \top} \mathcal{I}_{l} \tilde{x}_{b}^{r}\right) \\
& =\frac{1}{2}\left(\tilde{x}_{b}^{r \top} L\left(\alpha_{s}\right) \tilde{x}_{b}^{r}+\tilde{x}_{b}^{r \top} \mathcal{I}_{l} \tilde{x}_{b}^{r}\right) \geq 0,
\end{aligned}
$$

as candidate Lyapunov function, it is obtained

$$
\begin{aligned}
\left\langle\nabla V_{c}\left(\tilde{x}_{b}^{r}\right), f_{b l}\right\rangle & =-\tilde{x}_{b}^{r \top} L\left(\alpha_{s}\right) K_{c} L\left(\alpha_{s}\right) \tilde{x}_{b}^{r} \\
& -2 \tilde{x}_{b}^{r \top} L\left(\alpha_{s}\right) K_{c} \mathcal{I}_{l} \tilde{x}_{b}^{r}-\tilde{x}_{b}^{r \top} \mathcal{I}_{l} K_{c} \mathcal{I}_{l} \tilde{x}_{b}^{r}<0,
\end{aligned}
$$

where $f_{b l}=-K_{c}\left(L\left(\alpha_{s}\right) \tilde{x}_{b}^{r}+\tilde{x}_{b}^{r}\right)$. Indeed, the control law defined in the dynamic $x_{b}^{r}$ guarantees that the specific $x_{b, i}^{r}$ of all $\mathfrak{L}$ nodes designed as leaders converge to $x_{b}^{*}$. Hence, the consensus algorithm ensures that the followers also converge to $x_{b}^{*}$, because $L\left(\alpha_{s}\right) x_{b}^{r} \rightarrow \mathbf{0}$.

Now,it is possible to formulate the theorem that guaranties $\mathcal{A}$ be SPAS for hybrid system $\mathcal{H}$, i.e., the deal is to ensure that both proposed controls: the distributed control law for $x_{b}$ and the local control for $x$ stabilize the global system $\mathcal{H}$.

Theorem 1: Consider equilibrium $x_{e}$ with its associated vector $\lambda:=\left[\lambda_{1}, \lambda_{2}, . ., \lambda_{N}\right]$ ensuring Assumption 1, and a selected $T_{i}$ for each $i \in \mathcal{N}$, such that, Assumption 2 is satisfied. Consider an interconnected graph, $\mathcal{G}(\mathcal{N}, \mathcal{E})$ with $\mathfrak{L}$ leaders. Moreover, if there exist some matrices $P_{i}, Q_{i} \in \mathbb{R}^{n_{i}} \succ 0$ that satisfy Property 1 for each $i \in \mathcal{N}$, then $\mathcal{A}$ is SPAS for the hybrid system $\mathcal{H},(17)-(20)$.

Proof: The proof is based on singular perturbation theory applied to hybrid dynamical systems given in [29]. Therefore, it is straightforward from Proposition 1-4.

Remark 3: If all $T_{i}$ are sufficiently small the SPAS set $\mathcal{A}$ can be close to the SPAS set $\mathcal{A}_{0}:=\mathcal{A}_{0,1} \cup \mathcal{A}_{0,2} \cup$.. $\cup \mathcal{A}_{0, N}$ with

$$
\mathcal{A}_{i, 0}:=\left\{\xi_{i} \in \mathbb{H}_{i}: V_{i}\left(\xi_{i}\right)<0, \sigma_{i} \in \mathbb{K}_{i}, \tau=0\right\} \times R_{i} \mathbb{B}_{i} .
$$

In many practical situations, it is desirable that the current consensus achieved by the $x_{b, i}$ are weighted by the generation capacities on the batteries. In order to consider this fact, the Laplacian matrix $L\left(\alpha_{s}\right)$ can be redesigned, introducing the weight of every node $i$ in the microgrid, and thus, leading to the following corollary that establishes stability properties in this particular case.

Corollary 1: Consider that every node is weighted by $w_{i} \in[0,1]$, such that $\sum_{i \in \mathcal{N}} w_{i}=1$, being the Laplacian matrix $L\left(\alpha_{s} w\right)$, with $w=\operatorname{diag}\left\{w_{1}, w_{2}, . ., w_{N}\right\}$. Then, the desired consensus comes defined by the vector

$$
x_{b}^{*}=i_{d} w \mathbf{1}
$$

and the hybrid system, $\mathcal{H},(17)-(20)$ is modified by the following dynamic

$$
\dot{x}_{b}^{r}=-K_{c}\left(L\left(\alpha_{s}\right) w x_{b}^{r}+\alpha_{s} \mathcal{I}_{l} \tilde{x}_{b}^{r}\right)-\alpha_{s}\left(x_{\gamma}-x_{e, \gamma}\right),
$$

driving to a new hybrid scheme $\mathcal{H}_{w}(w)$.

Then $\mathcal{A}$ is SPAS for the hybrid system $\mathcal{H}_{w}$.

The proof is direct from Theorem 1 proof.

\section{Simulations}

This section is depicted to validate in simulation the main result given in Theorem 1 . Here, it is considered a microgrid composed by $N=5$ DGs and batteries (or nodes) and $L=4$ PCLs. The parameter values are given in Table I. The voltage of the PCC is $V_{b u s}=48 \mathrm{~V}$ and the batteries in supply mode are connected by boost converters, with the following parameters for all nodes $i$ : an inductance of $L_{c, i}=470 \mu \mathrm{H}$, a capacitance of $C_{c, i}=$ $600 \mu \mathrm{F}$ and a dissipative load of $R_{c, i}=3 \Omega$. The state is $x_{i}=\left[i_{L, i}, v_{C, i}\right]^{\top}$, with $i_{L, i}$, the current through the inductance $L_{i}$ and $v_{C, i}$ the tension over the capacitance $C_{i}$. The switched space state model (5) is

$$
A_{0, i}=\left[\begin{array}{cc}
\frac{R_{c, i}}{L c, i} & 0 \\
0 & -\frac{1}{R_{l, i} C_{c, i}}
\end{array}\right], \quad A_{1, i}=\left[\begin{array}{cc}
\frac{R_{c, i}}{L c, i} & -\frac{1}{L_{c}} \\
\frac{1}{C_{c}} & -\frac{1}{R_{l, i} C_{c, i}}
\end{array}\right],
$$

and

$$
B_{0, i}=B_{1, i}=\left[\begin{array}{c}
\frac{V_{i n, i}}{L c, i} \\
\frac{V_{b u s}}{R_{l, i} C_{c, i}}
\end{array}\right]
$$

\begin{tabular}{|c|c|c|c|c|c|}
\hline \multicolumn{7}{|c|}{ DGs } \\
\hline$i$ & 1 & 2 & 3 & 4 & 5 \\
\hline$i_{g, i}(\mathrm{~A})$ & 8 & 12 & 15 & 8 & 7 \\
\hline$i$ & 1 & 2 & 3 & 4 & 5 \\
\hline$V_{i n, i}(\mathrm{~V})$ & 60 & 58 & 35 & 61 & 43 \\
\hline$R_{l, i}(\Omega)$ & 2 & 1.34 & 3.67 & 2.34 & 3 \\
\hline \multicolumn{7}{|c|}{ PCL } \\
\hline$j$ & 1 & 2 & 3 & & \\
\hline$P_{w, d, j}(\mathrm{KW})$ & 4 & 3 & 2.4 & & \\
\hline
\end{tabular}

TABLE I: Microgrid data.

The graph $\mathcal{G}(\mathcal{N}, \mathcal{E})$ of the microgrid is composed by 5 nodes $\left(\mathcal{N}_{i}\right)$ and every node $i$ is connected with its neighbourhood. Likewise, there is $\mathfrak{L}=1$ leader, which is node $\mathcal{N}_{1}$, as shown in Fig. 4.

The matrices $P_{i}$ and $Q_{i}$ are computed satisfying Property 1. Applying [2, Theorem 2], which allows achieving a trade off between any performance level and switching reduction in the transient time, it is chosen $\eta_{i}=0.32$ for all nodes $i$. Moreover, the minimum dwell times, $T_{i}^{*}$, mentioned in Property 2 are computed considering a 


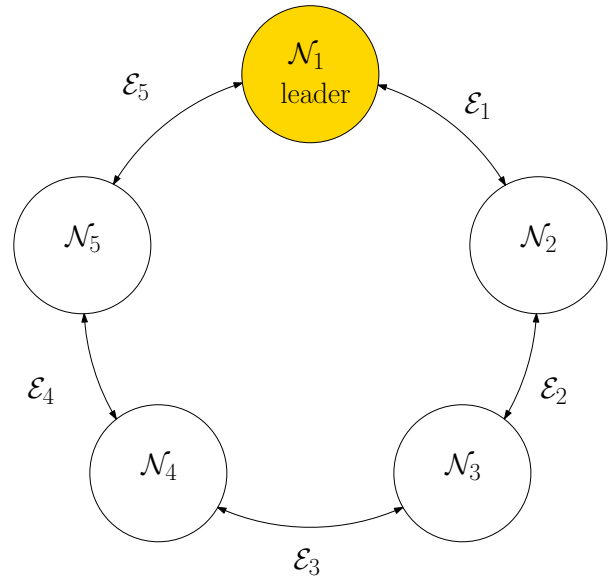

Fig. 4: Microgrid graph.

maximum chattering of $i_{L, i}$ equal to $2 A$ and a maximum chattering of $v_{C, i}$ equal to $5 \mathrm{~V}$, giving $T_{1}^{*}=0.0037 \mathrm{~s}$, $T_{2}^{*}=0.0195 \mathrm{~s}, T_{3}^{*}=0.0014 \mathrm{~s}, T_{4}^{*}=0.0003 \mathrm{~s}$ and $T_{5}^{*}=$ $0.0022 \mathrm{~s}$, which satisfy (14). Thus, $T^{*}=0.0003 \mathrm{~s}$. Hence, it is chosen $T_{d w}=10 \mu \mathrm{s}$ and $K_{c, i}=3 \cdot 10^{6}$ for satisfying Assumption 2.

The validation of Theorem 1 is performed from the following scenarios:

a) Scenario 1: Initially, all batteries are in supply mode. The battery of node $\mathcal{N}_{3}$ fails at $0.006 \mathrm{~s}$ and it is again connected at $0.032 \mathrm{~s}$. Likewise, the battery of node $\mathcal{N}_{2}$ changes from supply mode to loading mode at $0.024 \mathrm{~s}$. Finally, there is a load, $\mathcal{L}_{1}$, which is disconnected from the microgrid at $0.016 \mathrm{~s}$. These connections/disconnections are shown in Fig. 5. Likewise, it is performed in Fig. 6, the evolutions of this scenario. Note as the reference current of the leader, $i_{b}^{r}$, is quickly adapted according to the demanded current changes, occurred by plug in play of any DG, battery or load or any change of the battery mode. Likewise, the output current of the converters in supply mode asymptotically converge to a neighbourhood of their equilibriums. Note that the $i_{b}$ of the nodes in loading mode or in failure are not concerned by the consensus objective.
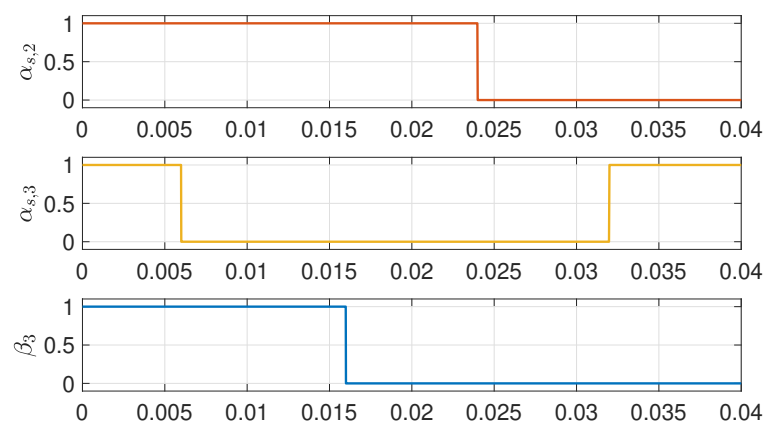

Fig. 5: From the top, the following evolutions of the exogenous signals, $\alpha_{s, 2}, \alpha_{s, 3}$ and $\beta_{4}$.
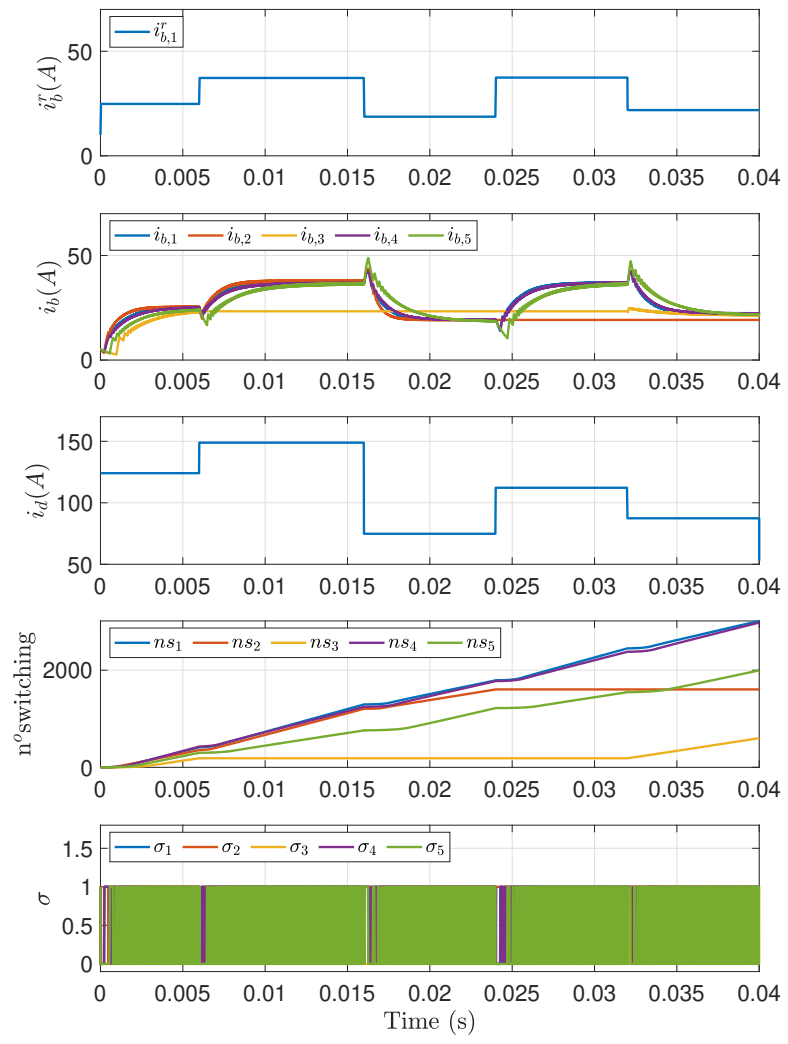

Fig. 6: From the top, the following evolutions: current reference of the leader $\mathcal{N}_{1}, i_{b, i}^{r}$, output currents of each node, $i_{b}$, demanded current $i_{d}$, number of switching of each node and discrete input variables $\sigma_{i}$.

b) Scenario 2: This scenario illustrates Corollary 1 and the fact that some connections can fail. Initially, all batteries are in supply mode. Then, connection $\mathcal{E}_{3}$ fails at $0.01 \mathrm{~s}$ and then $\mathcal{E}_{1}$ fails at $0.025 \mathrm{~s}$. Note as the currents, $i_{b, i}$, converge to their references, which are proportional to the input source $V_{i n, i}$ of each node $i$. It is highlighted that the current balancing (1) is hold. Moreover, the hybrid system $\mathcal{H}_{w}$ is robust with respect to communication lost between nodes.

\section{Conclusions}

This work provides a novel paradigm based on HDS and MAS theory for modelling and controlling DCbus microgrids in islanded mode. Indeed, two control levels are identified. On one hand, an inner control loop drives DC-DC converters, considering the hybrid nature: the continuous-time signals (voltages and currents) and discrete-time signals (state of the switches). This control is based on HDS theory, which allows obtaining some advantages in terms of switching reduction during transient times and avoiding current peaks in start up. On the other hand, the outer control, which is a leaderfollower consensus control, designed from MAS theory, 

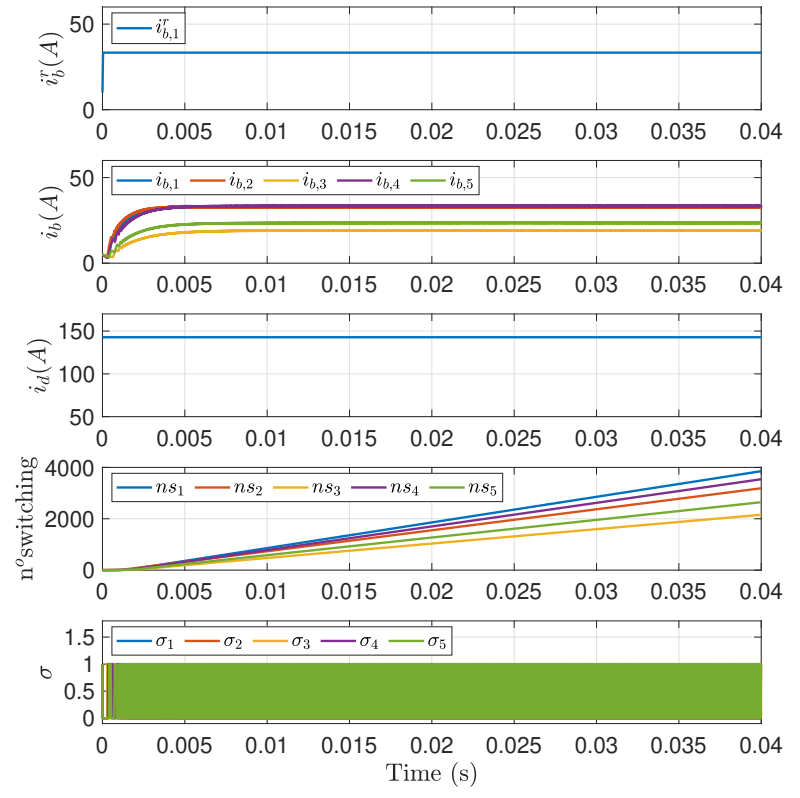

Fig. 7: From the top, the following evolutions: current reference of the leader $\mathcal{N}_{1}, i_{b, i}^{r}$, output currents of each node, $i_{b}$, demanded current $i_{d}$, number of switching of each node and discrete input variables $\sigma_{i}$, when connection $\mathcal{E}_{3}$ fails at $0.01 \mathrm{~s}$ and $\mathcal{E}_{1}$ fails at $0.025 \mathrm{~s}$.

leads to a distributed control which ensures robustness with respect to plug in play of the batteries, sources or loads, connection failures and parameter variations. Stability properties are guaranteed considering time-scale separation, forced by tuning the consensus parameters. Indeed, the outer control loop will evolve faster than the inner control loop, in such away to have a regulation problem in the inner loop. Singular perturbation theory is employed for guaranteeing that attractor $\mathcal{A}$ is SPAS. Some simulations validate the main result.

As future work, it is envisioned to consider another control loop to manage the functioning mode of the batteries (loading mode or source mode), as well as, to obtain experimental results.

\section{ACKNOWLEDGMENTS}

This work has been partially funded under grant "HISPALIS" ANR-18-CE40-0022-01.

\section{REFERENCES}

[1] C. Albea Sanchez, G. Garcia, S. Hadjeras, W.P.M.H. Heemels, and L. Zaccarian. Practical stabilisation of switched affine systems with dwell-time guarantees. IEEE Trans. on Automatic Control, 2019.

[2] C. Albea Sanchez, G. Garcia, and L. Zaccarian. Hybrid dynamic modeling and control of switched affine systems: application to DC-DC converters. In IEEE Conference on Decision and Control, pages 2264-2269, Osaka, Japan, 2015.

[3] C. Albea Sanchez, O. Lopez Santos, D.A. Zambrano Prada, F. Gordillo, and G. Garcia. On the practical stability of hybrid control algorithm with minimum dwell time for a DCAC converter. IEEE Trans. on Control Systems Technology, 2018.
[4] S. Anand, B. G Fernandes, and J. Guerrero. Distributed control to ensure proportional load sharing and improve voltage regulation in low-voltage DC microgrids. IEEE Trans. on Power Electronics, 28(4):1900-1913, 2013.

[5] A. Antonopoulos, L. Angquist, and H.P. Nee. On dynamics and voltage control of the modular multilevel converter. In IEEE European Conference on Power Electronics and Applications (EPE), pages 3353-3362. IEEE, 2009.

[6] Y. Che, Z. Yang, and K.W.E. Cheng. Construction, operation and control of a laboratory-scale microgrid. In IEEE Conference on Power Electronics Systems and Applications., pages $1-5,2009$.

[7] J.F. Chen and C.L. Chu. Combination voltage-controlled and current-controlled pwm inverters for ups parallel operation. IEEE Trans. on Power Electronics, 10(5):547-558, 1995.

[8] M. Cucuzzella, S. Trip, C. De Persis, X. Cheng, A. Ferrara, and A. van der Schaft. A robust consensus algorithm for current sharing and voltage regulation in DC microgrids. IEEE Trans. on Control Systems Technology, (99):1-13, 2018.

[9] K. De Brabandere, B. Bolsens, J. Van den Keybus, A. Woyte, J. Driesen, and R. Belmans. A voltage and frequency droop control method for parallel inverters. IEEE Trans. on power electronics, 22(4):1107-1115, 2007.

[10] G. S. Deaecto, J. C. Geromel, F.S. Garcia, and J.A. Pomilio. Switched affine systems control design with application to DCDC converters. IET control theory \& applications, 4(7):12011210, 2010.

[11] D.V. Dimarogonas, P. Tsiotras, and K.J. Kyriakopoulos. Leader-follower cooperative attitude control of multiple rigid bodies. Systems \& Control Letters, 58(6):429-435, 2009.

[12] R. Goebel, R. Sanfelice, and A.R. Teel. Hybrid dynamical systems. IEEE Control Systems Magazine, 29(2):28-93, April 2009.

[13] R. Goebel, R.G. Sanfelice, and A.R. Teel. Hybrid Dynamical Systems: modeling, stability, and robustness. Princeton University Press, 2012.

[14] J. M. Guerrero, J.C. Vasquez, J. Matas, L. Garcia De Vicuna, and M. Castilla. Hierarchical control of droop-controlled AC and DC microgrids - a general approach toward standardization. IEEE Trans. on Industrial Electronics, 58(1):158-172, 2011.

[15] H. Han, X. Hou, J. Yang, J. Wu, M. Su, and J.M. Guerrero. Review of power sharing control strategies for islanding operation of AC microgrids. IEEE Trans. on Smart Grid, 7(1):200$215,2016$.

[16] D. He, D;and Shi and R. Sharma. Consensus-based distributed cooperative control for microgrid voltage regulation and reactive power sharing. In IEEE PES Innovative Smart Grid Technologies Conference Europe (ISGT-Europe), pages 1-6, 2014.

[17] L. Hetel and E. Fridman. Robust sampled-data control of switched affine systems. IEEE Trans. on Automatic Control, 58(11):2922-2928, 2013.

[18] B.G. Kamdem and E. Shittu. Optimal commitment strategies for distributed generation systems under regulation and multiple uncertainties. Renewable and Sustainable Energy Reviews, 80:1597-1612, 2017.

[19] A. Kwasinski and C.N. Onwuchekwa. Dynamic behavior and stabilization of DC microgrids with instantaneous constantpower loads. IEEE Trans. on Power Electronics, 26(3):822$834,2011$.

[20] R.H. Lasseter. Microgrids and distributed generation. Journal of Energy Engineering, 133(3):144-149, 2007.

[21] X. Lu, J.M. Guerrero, K. Sun, and J.C. Vasquez. An improved droop control method for DC microgrids based on low bandwidth communication with DC bus voltage restoration and enhanced current sharing accuracy. IEEE Trans. on Power Electronics, 29(4):1800-1812, 2014.

[22] A. Martinelli, A. La Bella, and R. Scattolini. Secondary control strategy for DC islanded microgrids operations. In IEEE European Control Conference (ECC), 2019.

[23] L. Martínez-Salamero, G. Garcia, M. Orellana, C. Lahore, B. Estibals, C. Alonso, and C.E. Carrejo. Analysis and design of a sliding-mode strategy for start-up control and voltage regulation in a buck converter. IET Power Electronics, 6(1):52-59, 2013.

[24] R.D. Middlebrook and S. Cuk. A general unified approach to 
modelling switching-converter power stages. In IEEE Power Electronics Specialists Conference, pages 18-34, 1976.

[25] R. Olfati-Saber, J.A. Fax, and R.M. Murray. Consensus and cooperation in networked multi-agent systems. Proceedings of the IEEE, 95(1):215-233, 2007.

[26] R. Olfati-Saber and R.M. Murray. Graph rigidity and distributed formation stabilization of multi-vehicle systems. In IEEE Decision and Control, (CDC), volume 3, pages 29652971,2002

[27] R. Olfati-Saber and R.M. Murray. Consensus problems in networks of agents with switching topology and time-delays. IEEE Trans. on Automatic Control, 49(9):1520-1533, 2004.

[28] J. Rocabert, A. Luna, F. Blaabjerg, and P. Rodriguez. Control of power converters in AC microgrids. IEEE Trans. on Power Electronics, 27(11):4734-4749, 2012.

[29] R.G Sanfelice and A.R Teel. On singular perturbations due to fast actuators in hybrid control systems. Automatica, 47(4):692-701, 2011.

[30] A. Sferlazza, C. Albea Sanchez, L. Martínez-Salamero, G. Garcia, and C. Alonso. Min-type control strategy of a DCDC synchronous boost converter. IEEE Trans. on Industrial Electronics, 2019.

[31] H. Sira-Ramírez and R. Silva-Ortigoza. Control design techniques in power electronics devices. Springer Science \& Business Media, 2006.

[32] K. Siri, C.Q. Lee, and T.E. Wu. Current distribution control for parallel connected converters. IEEE Trans. on Aerospace and Electronic Systems, 28(3):829-840, 1992.

[33] T. A.F. Theunisse, J. Chai, R.G. Sanfelice, and W.P. Heemels. Robust global stabilization of the DC-DC boost converter via hybrid control. IEEE Trans. on Circuits and Systems I: Regular Papers, 62(4):1052-1061, 2015.

[34] M. Tucci, S. Riverso, J.C. Vasquez, J.M. Guerrero, and G. Ferrari-Trecate. A decentralized scalable approach to voltage control of DC islanded microgrids. IEEE Trans. on Control Systems Technology, 24(6):1965-1979, 2016.

[35] Y. Xu and Z. Li. Distributed optimal resource management based on the consensus algorithm in a microgrid. IEEE Trans. on Industrial Electronics, 62(4):2584-2592, 2015.

[36] D. Zonetti, A. Saoud, A. Girard, and L. Fribourg. A symbolic approach to voltage stability and power sharing in timevarying DC microgrids. In IEEE European Control Conference (ECC), 2019. 